\title{
Management of the frozen shoulder
}

This article was published in the following Dove Press journal:

Orthopedic Research and Reviews

7 October 2014

Number of times this article has been viewed

\section{Suzanne Margaretha van de Laar \\ Peer van der Zwaal \\ Department of Orthopaedic Surgery and Trauma, Medical Center Haaglanden, The Hague, the Netherlands}

Correspondence: Peer van der Zwaal PO Box 432, 250 I CK, The Hague, the Netherlands

Tel +3 I 65203885 I

$\mathrm{Fax}+31703302839$

Email p.van.der.zwaal@mchaaglanden.nl
Abstract: Frozen shoulder is a very common condition with a prevalence of $2 \%-5 \%$ in the general population. Decrease in joint volume as a result of fibrosis and hyperplasia of the joint capsule leads to painful and restricted glenohumeral motion. Frozen shoulder is a selflimiting disease with a chronic character, and is mostly treated in a primary care setting. In this review, we set out to address the current evidence-based literature on management of this disabling disease using a PubMed search. Many non-surgical and surgical therapeutic options are described, including supervised neglect, intra-articular corticosteroid injections, physical therapy, manipulation under anesthesia, capsular distension, and arthroscopic capsular release. In the literature, the long-term outcome shows a significant decrease in pain and improvement of shoulder function for all treatment modalities without clear evidence of superiority of one over the other. This possibly indicates that a self-limiting character is the most important factor in the course of the disease. Management of frozen shoulder is primarily conservative. Supervised neglect is combined with analgesia and stretching exercises as the pain subsides. In the early painful phase, intra-articular corticosteroid injections are recommended for pain relief. When the patient has persistent pain and glenohumeral stiffness after adequate conservative treatment, invasive options can be considered, like arthroscopic capsular release, manipulation under anesthesia, or capsular distension.

Keywords: frozen shoulder, adhesive capsulitis, treatment, pathophysiology

\section{Introduction}

Frozen shoulder, also known as adhesive capsulitis, is a disabling disease. It is characterized by shoulder pain and limitations of both active and passive range of movement in all directions. Limitation of glenohumeral movement is due to decreased intra-articular volume. It is the result of fibrosis and thickening of the joint capsule and adherence to the humeral head. Frozen shoulder is self-limiting in almost all cases. The natural course takes 12-42 months before resolution. ${ }^{1}$ Fifteen percent of the patients experience long-term disability as a result of chronic loss of shoulder mobility. ${ }^{2,3}$ Pain is less prominent in this group of patients. Clinicians' opinions differ on the evaluation and treatment of frozen shoulder. Many treatment regimens are described in the literature. In this review, we set out to address the current evidence-based literature on management of this disabling disease.

\section{Search methods}

The PubMed database was screened for papers involving the following keywords "frozen shoulder", "adhesive capsulitis", "etiology", "management", "therapy", 
and "pathophysiology". For clinical papers, we focused on randomized controlled trials published in peer-reviewed journals.

\section{Epidemiology}

The prevalence of frozen shoulder is $2 \%-5 \%$ in the general population, with a peak in the fifth and sixth decades of life. ${ }^{2}$ A frozen shoulder is rarely seen in patients under the age of 40 years. Women are more affected then men. Twins have a 2-3-fold higher risk once one of them has developed a frozen shoulder. There is no known genetic predisposition., ${ }^{2,4}$ The non-dominant arm is slightly more affected then the dominant arm. ${ }^{5,6}$ Once a patient has experienced an episode of frozen shoulder, the risk of recurrence on the contralateral side is $6 \%-17 \%$ within 5 years. Recurrence in the same shoulder is rare. ${ }^{7}$ There is no evidence in the literature that specific subgroups in the population would have an increased risk of developing a frozen shoulder compared with others. Speculations about a "frozen shoulder personality" are not proven in the literature. ${ }^{8}$

\section{Pathophysiology}

The pathophysiology of frozen shoulder is not completely clear. A commonly accepted theory is that fibrosis causes thickening of the glenohumeral joint capsule, which becomes more tight. Adhesion of the capsule to itself and to the neck of the humerus causes obliteration of the axillary fold, reduction in joint volume, minimal presence of synovial fluid, and restricted glenohumeral movement.

Frozen shoulder has been described by many authors in the past, and is known by almost the same number of names. In 1872, this condition was described by Duplay as "peri-artritis". 9 In 1934, Codman introduced the term "frozen shoulder" as a disease of slow onset, with inability to sleep on the affected side due to severe pain and stiffness in all directions without radiologic abnormalities. ${ }^{10}$ In 1945, Neviaser named the condition "adhesive capsulitis". He was the first to combine observations from a cadaver study with histologic analysis, and reported thickening of the joint capsule and adherence of the capsule to the humeral head with an unaffected bursa. ${ }^{11}$ He suggested that this is due to a chronic inflammatory process. This theory was later contradicted by Lundberg, and Bunker and Anthony, who described a primary pathology of fibrosis, making the morphology of frozen shoulder the same as Dupuytren's contracture. They found no significant number of inflammatory cells and no synovial involvement. ${ }^{12,13}$ There is still no consensus in the literature as to whether inflammation is part of the pathophysiology or fibrosis is the basis of the histology of frozen shoulder.

Arthroscopy, magnetic resonance imaging (MRI), and cadaver studies have shown that the limitations in movement characteristic of frozen shoulder are explained by the macroscopic appearance of the affected anatomical structures. The rotator interval and the anterior capsule are the dominant focus of the abnormalities in primary frozen shoulder. Thickening of the anterosuperior capsule limits external rotation of the adducted arm, and the fibrous anteroinferior capsule will cause restrictions in external rotation in abduction. Thickening of the rotator interval compromises the coracohumeral ligament, causing limits in external rotation as well. ${ }^{14-16}$ In cadaver studies, contraction of the coracohumeral ligament is described, causing loss of external rotation in particular. ${ }^{17}$ In advanced stages, internal rotation is restricted, and is caused by tightening of the posterosuperior capsule.

In 1969, Lundberg made a distinction between primary and secondary frozen shoulder. In most cases, frozen shoulder develops primarily without an underlying disease. In secondary frozen shoulder, association with trauma to the shoulder, such as proximal humeral fractures, soft tissue injuries, subacromial bursitis, and shoulder surgery is described. A subset of patients has a systemic type of frozen shoulder. Diabetes mellitus, with a prevalence of $10 \%-20 \%$, is a risk factor for the development of frozen shoulder. Frozen shoulder in diabetic patients tends to be more severe and more resistant to therapy, and these patients have a greater risk of recurrence. ${ }^{18-21}$ Further, development of frozen shoulder is correlated with Dupuytren's disease, hyperthyroidism, and hypothyroidism. ${ }^{22}$ There is no evidence in the current literature indicating that development of a frozen shoulder is correlated with specific sports activities in the physically active population.

\section{Natural history}

Shaffer et al described the natural history of frozen shoulder in three clinical phases, ie, the freezing phase, the frozen phase, and the thawing phase (see Table 1). ${ }^{23}$

The freezing phase is characterized by pain in the shoulder at rest and with movement. Pain is worst at night due to capsular stretching and patients have difficulty sleeping. In this stage, the pain intensifies and the range of movement gradually decreases. Restrictions in external rotation occur first, followed by internal rotation and abduction. ${ }^{24}$ This stage takes 2-9 months before the second phase begins. Pain is less prominent in the frozen phase, and stiffness is the greatest complaint of patients in this stage of the disease. Daily 
Table I Natural history of frozen shoulder

Clinical phases
Freezing phase
Pain in the shoulder, worse at night
Stiffness
$2-9$ months
Frozen phase
Less pain
Stiffness, especially external rotation
4-12 months
Thawing phase
Gradual return of range of motion
5-24 months

Note: Data from Shaffer et al. ${ }^{23}$

activities like brushing hair or reaching for something in the cupboard cause difficulties. The pain at night-time slowly subsides and patients have fewer problems sleeping. This stage takes 4-12 months before the final stage sets in. The thawing phase is characterized by an absence of pain and gradual increase of glenohumeral motion over a period of 4-12 months. At the end, patients have regained their shoulder functionality and can perform normal daily activities. Because not all patients follow the described phases, clinical application of this classification can be difficult.

Neviaser and Neviaser described the frozen shoulder in four pathophysiologic stages based on arthroscopic findings. ${ }^{25}$ In the initial stage, there is formation of fibrinous inflammation in the axillary recess. Adhesions do not occur in this stage. Patients suffer from intense pain in the shoulder without loss of range of motion. The second stage is characterized by acute adhesive synovitis, and the joint capsule adheres to the humeral head and to itself. Pain is still severe and mobility is gradually restricted. In the third stage, fibrosis is more prominent than synovitis. This is the so-called maturation stage. The axillary fold is completely obliterated, causing pronounced restriction in motion. Pain can still be present but is not as intense as in the initial stage. The fourth and most chronic stage is marked by severe loss of motion caused by adhesions. Stretching the shoulder beyond the limits of these adhesions will cause pain, although pain has moved to the background in this stage of the disease.

A frozen shoulder will resolve spontaneously within 2-4 years in almost all patients. In a prospective study of 41 patients with 5-10 years of follow-up, Reeves concluded that $39 \%$ of patients had no remaining symptoms, $54 \%$ had clinical limitations without functional disability, and $7 \%$ of patients continued to have restrictions in range of motion. ${ }^{1}$ Shaffer et $\mathrm{al}^{23}$ found $50 \%$ of 61 patients to have some level of functional limitations or pain 7 years after onset of the disease. However, for the most part, the persisting limitations of movement after frozen shoulder are not functionally limiting. ${ }^{26,27}$ Hand et al ${ }^{5}$ studied 269 patients with frozen shoulder and found that $41 \%$ had ongoing symptoms, of which $94 \%$ were mild, and only $6 \%$ had severe long-term symptoms with pain and functional loss. Patients with very severe symptoms at onset of the disease had the highest likelihood of ongoing symptoms at a later stage. ${ }^{6}$

\section{Clinical presentation}

In the shoulder, there is a perfect compromise between mobility and stability. However, due to its wide range of motion and osseous anatomy, there is a relatively high risk of dislocation in the shoulder compared with other joints. Stability is mainly ensured by the rotator cuff unit, glenoid labrum, glenohumeral ligaments, and joint capsule. This is the reason that stiffness of the shoulder is mainly due to soft tissue problems rather than other factors, like osteophytes or loss of congruity.

A painful shoulder with restricted mobility is a common presentation in daily orthopedic practice. Careful evaluation of symptoms through history and physical examination is crucial to differentiate frozen shoulder from acromioclavicular joint, subacromial pain, and biceps tendon or rotator cuff problems.

Frozen shoulder is characterized by severe shoulder pain and loss of both active and passive movement in all directions. Specifically, loss of external rotation is typically present in a frozen shoulder. Patients experience loss of function in activities of daily living and difficulty sleeping on the affected side. The deltoid and supraspinatus muscles may be atrophic due to disuse, followed by the infraspinatus, subscapularis, and teres minor muscles. As a result of disuse and atrophy of these muscles, the kinematics of the joint will change, leading to decreased functionality. Translation of the humeral head will be reduced, mainly as a result of tightness of the inferior glenohumeral ligament. Also, the scapula will show lateral rotation in relation to glenohumeral rotation during elevation of the shoulder at an early stage. ${ }^{28,29}$

Although frozen shoulder cannot be seen on X-ray, radiography is necessary to rule out other conditions like osteoarthritis or a bone tumor. Frozen shoulder secondary to fracture or surgery can be observed. On MRI, capsular thickening and contraction of the axillary recess may be present. Nevertheless, MRI is not a diagnostic standard as the diagnosis is based on clinical presentation. Biochemical or hematological investigation can be performed to rule out secondary causes if indicated. 


\section{Treatment}

Management of frozen shoulder can be done in the primary care setting. When the pain and restricted range of motion do not resolve, the patient must be referred to an orthopedic surgeon for additional treatment and follow-up.

Because frozen shoulder is a predominantly self-limiting disease, supervised neglect can be sufficient, and with time the frozen shoulder will resolve. Immobilization carries a high risk of additional stiffness, so patients are advised to keep using the affected arm within pain limits. Patients should be educated that the disease passes through the described phases, and that it will take months, sometimes even years, before clinical improvement will occur. Pain relief and a more rapid return of range of movement are the goals of treatment, which depend on the phase of the disease. In the freezing phase, treatment will be focused on pain relief and retention of range of motion. In the frozen phase, pain management is less prominent and gaining range of motion will be the main goal.

Primarily, a non-surgical treatment is chosen. However, when a patient has refractory symptoms after adequate conservative treatment, surgical options can be considered. Specific treatment options are discussed below. There is still no evidence-based consensus for the treatment of this painful shoulder condition. In a systematic review by Rookmoneea et al on the effectiveness of interventions in management of primary frozen shoulder, no definitive conclusions could be drawn because of poor methodological quality. ${ }^{30}$ Underlying causes like diabetes mellitus, thyroid disease, or non-union of humeral fractures, should be treated to optimize the healing of frozen shoulder. If the frozen shoulder is secondary to a rotator cuff tear, bursitis, or labral tear, diagnosis and treatment will be difficult as more problems are present.

\section{Non-surgical treatment Physical therapy}

Physical therapy and supervised home exercises are considered important in the non-surgical treatment of shoulder pain and frozen shoulder, although there is no hard evidence in the literature. ${ }^{31}$ Moderated stretching is most important in the physical therapy program. Pain and strengthening will cause discomfort for the patient without improved range of motion and should be avoided. Gentle stretching exercises within the limits of pain can achieve greater mobility then aggressive stretching and strengthening. ${ }^{28,32}$ Stretching exercises of the shoulder capsule are performed in a controlled fashion. For the inferior capsule, the patient is in a seated position and the elbow and forearm are placed on a table (Figure 1A).
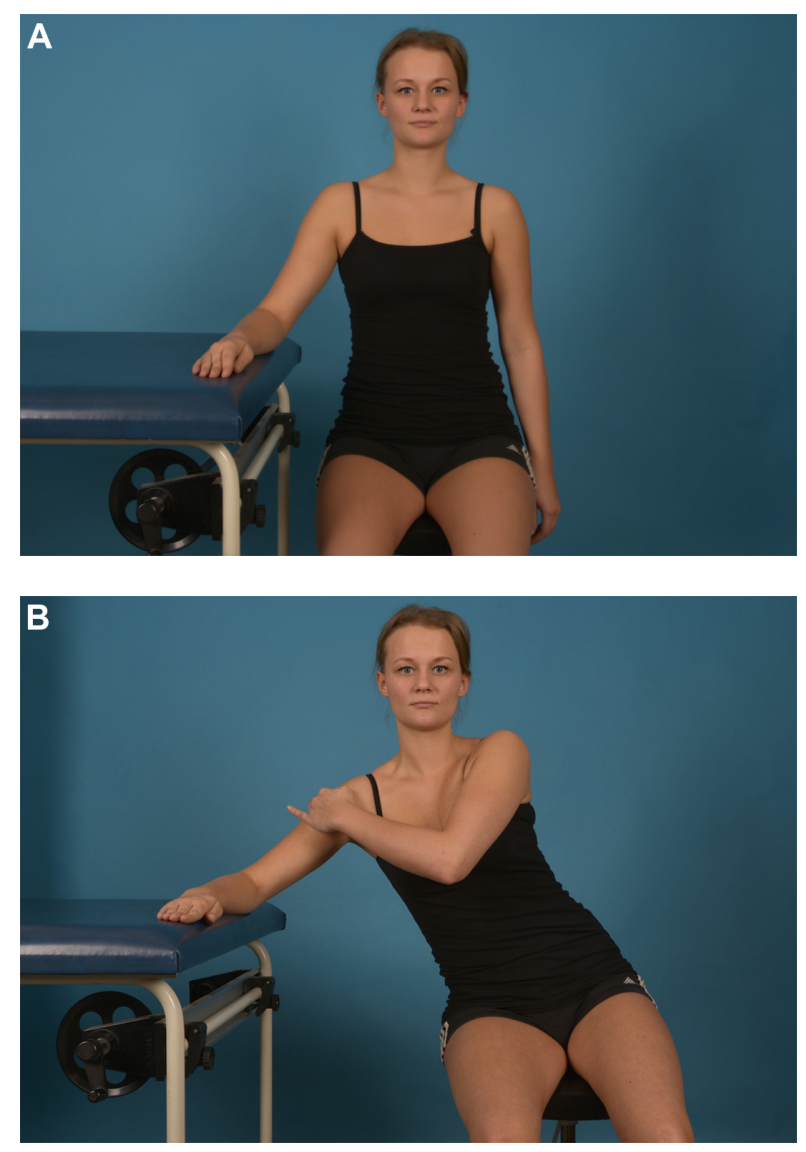

Figure I Stretching exercise of the inferior glenohumeral capsule.

Notes: (A) The patient is in a seated postion, and the elbow and forearm are placed on a table. (B) The body slides away from the table, while the contralateral hand stabilizes the shoulder and produces a downward force.

The patient's body now slowly slides away from the table, while the contralateral hand stabilizes the shoulder and produces a downward force stretching the inferior capsule (Figure 1B). For the anterior capsule, the patient is in a standing position with the elbow in 90 degrees of flexion and 0 degrees of abduction. The ventral forearm is placed against a doorframe (Figure 2A). Now the patient slowly turns the body away from the doorframe, producing an external rotation force and stretching of the anterior capsule (Figure 2B). Stretching exercise of the posterior capsule: the patient is in the lateral decubitus position. The arm is in 90 degrees of forward flexion and the elbow in 90 degrees of flexion (Figure 3A,B). The contralateral hand grasps the wrist of the affected arm and gently produces an internal rotation force, thereby stretching the posterior capsule (Figure 3B). The stretching maneuvers are maintained for 10 seconds within pain limits, with 10-15 repetitions. The sets of exercises should be performed twice a day.

A blinded, randomized controlled trial in 75 patients compared the difference in outcome of exercises in a group exercise class, individual physical therapy, and home 

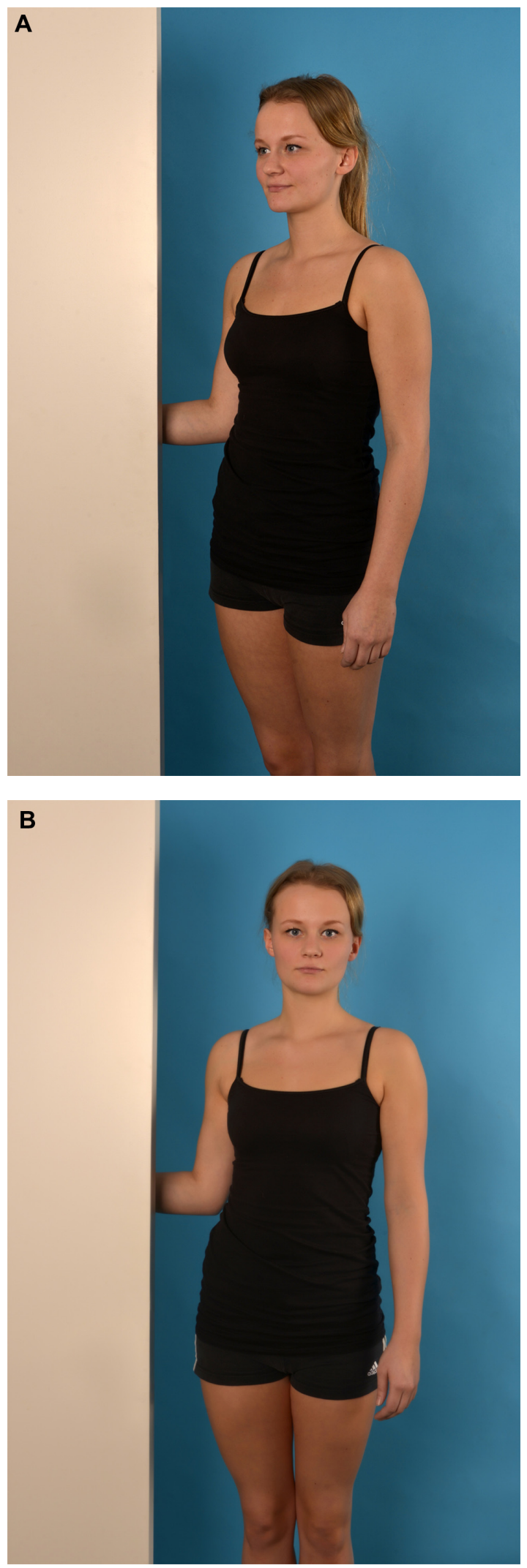

Figure 2 Stretching exercise of the anterior glenohumeral capsule.

Notes: (A) The patient is in a standing position with the elbow in 90 degrees of flexion and 0 degrees of abduction. The ventral forearm is placed against a doorframe. (B) Now the patient slowly turns their body away from the doorframe, producing an external rotation force.
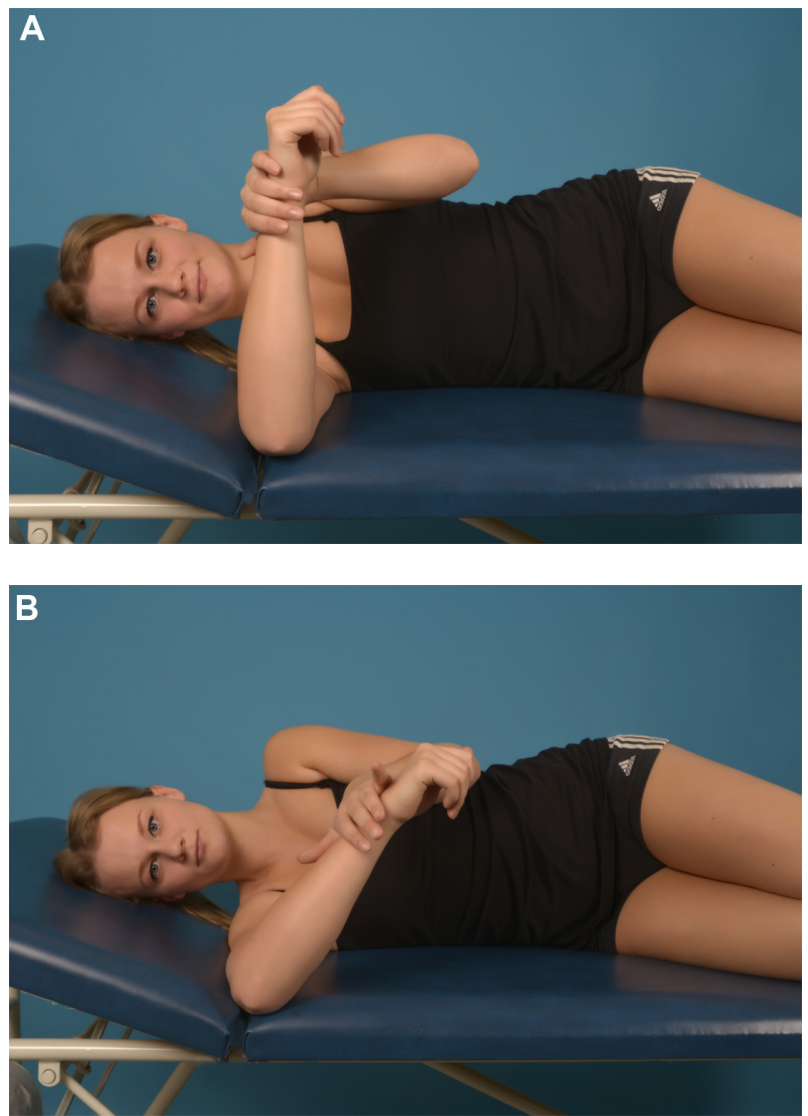

Figure 3 Stretching exercise of the posterior glenohumeral capsule.

Notes: (A) The patient is in the lateral decubitus position. The arm is in 90 degrees of forward flexion and the elbow in 90 degrees of flexion. (B) The contralateral hand grasps the wrist of the affected arm and gently produces an internal rotation force.

exercises. The group exercise class showed the most rapid recovery within 1 year. ${ }^{33}$

\section{Medical treatment}

Non-steroidal anti-inflammatory drugs, both oral and intraarticular, are the main agents used, although they do not have long-term positive effects. Several studies in small numbers of patients have shown positive effects of oral glucocorticoids (eg, prednisolone $30 \mathrm{mg}$ once daily for 3 weeks). This will reduce pain and improve range of motion, although the effect will not last longer than a few weeks.

Buchbinder et al performed a meta-analysis of five trials representing a relatively small number of patients comparing steroids versus placebo (two trials), oral versus intra-articular steroids (one trial), oral steroids versus no treatment (one trial), and manipulation under anesthesia (MUA) versus intraarticular steroid injection with or without oral steroids (one trial). ${ }^{32}$ Their meta-analysis showed that steroids can induce short-term pain relief but the effects are not maintained beyond 6 weeks. No major adverse effects were reported. 
In daily practice, oral glucocorticoids do not have a prominent place in the management of frozen shoulder because of the short period of positive effects and their potential for serious adverse effects, such as osteonecrosis. ${ }^{34}$

\section{Intra-articular infiltration}

Intra-articular corticosteroid injections can induce shortterm pain relief and improvement in range of motion. This effect usually only lasts for a maximum of 6 weeks and is most effective when given in an early stage of the disease..$^{35,36}$ Intra-articular injections are most effective in reducing synovitis when given in the early stage. When the frozen shoulder has been present for a longer period of time and is more severe, the effect of infiltration on synovitis is limited. Because the joint space is obliterated, ultrasound may be useful to ensure correct placement of the injection. No more than three injections should be given, to avoid chondrolysis. ${ }^{37}$ The effects of medical treatment are temporary, and long-term follow-up studies have not shown improvement in pain and return of range of motion. ${ }^{38-42}$

In a randomized controlled trial, Carette et al studied 93 patients with frozen shoulder who had symptoms for more than 1 year. ${ }^{43}$ Patients were randomized to one of four treatment groups: intra-articular corticosteroid infiltration performed under fluoroscopy followed by physical therapy; intra-articular corticosteroid infiltration alone; intra-articular saline infiltration followed by supervised physical therapy; or intra-articular saline infiltration alone. All patients performed simple exercises at home. At 1 year of follow-up, all four groups had improved to a similar degree with respect to pain and function. Groups 1 and 2 showed a statistically significant rapid improvement, indicating the effectiveness of a single intra-articular injection with corticosteroids combined with simple home exercises in treating frozen shoulder. These authors speculated that addition of supervised physical therapy could achieve more rapid improvement in range of motion in the affected shoulder. Physical therapy without corticosteroid infiltration had results similar to those of saline infiltration alone without physical therapy. The authors concluded that physical therapy alone is of limited efficacy in treating frozen shoulder.

\section{Ultrasound, laser, and other non-surgical options}

A number of heating modalities have been suggested for the treatment of frozen shoulder to enhance the effect of exercises by relaxing the muscles and a positive influence on pain. A review in 2013 analyzed 39 studies of the effectiveness of non-surgical treatment modalities for frozen shoulder. ${ }^{44}$ They found that low-level laser therapy can be used for pain relief and improvement of shoulder function, but it will not improve range of motion. Electrotherapy can be used for short-term pain relief. Continuous passive motion can provide short-term relief of pain but does not increase function or range of motion. Deep heat can provide pain relief and improve range of motion. Ultrasound is not recommended for relief of pain or improvement of function or range of motion. Other therapies are described in low-level trials, such as acupuncture and supracapsular nerve blocks. ${ }^{45-47}$ These therapies are considered experimental because of a lack of evidence and experience with the therapy.

\section{Surgical treatment}

Surgical treatment should only be considered in patients who are refractory to adequate conservative therapy. There is no high-level evidence regarding the optimal time frame for referral. Generally, surgical treatment can be considered in patients without improvement after 12 months of conservative therapy and with severe disabling symptoms. If progress without surgical intervention is made, the next step should be postponed. Patients who do not gain full function of the shoulder after therapy could have an underlying disease, like rotator cuff pathology or osteoarthritis, and this should be diagnosed. Surgical options are joint MUA, arthroscopic capsular release, and capsular distension.

\section{Manipulation under anesthesia}

In MUA, the patient is brought under general anesthesia and the surgeon gradually abducts the arm in the scapular plane, rupturing the inferior capsule. ${ }^{48}$ External rotation and external rotation in abduction will follow. The lever arm produced by the surgeon should be short to prevent humeral fracture. Rotator cuff tears and glenohumeral dislocation could also occur as iatrogenic injuries. For this reason, MUA should be avoided in patients with osteoporosis, osteopenia, or a history of shoulder instability. Other contraindications include a shoulder prosthesis and previous open shoulder instability repair. Adhesions are disrupted and range of motion is restored. This intervention should be followed by physical therapy to maintain the range of motion. MUA has been used successfully, but there is no high-level evidence showing an advantage when compared with the natural history of frozen shoulder. ${ }^{49-51}$ A disadvantage of MUA is the inability to visualize the joint capsule, such that the surgeon cannot evaluate whether all areas of the contracted capsule are adequately addressed. In a randomized controlled trial 
including 125 patients, no difference was found between MUA followed by home exercises versus home exercises alone. $^{52}$

\section{Arthroscopic capsular release}

Arthroscopic capsular release is our preferred surgical option. This treatment is more invasive then MUA, but is also more controlled. The surgeon can control which part of the capsule is released. During arthroscopy, areas with synovitis and contraction are identified. Synovectomy is performed, followed by contracture release of the rotator interval, the anterosuperior, inferior and posterosuperior capsule.

A combined anesthetic technique is preferred for adequate postoperative pain relief, and allows the patient to start physical therapy exercises immediately. The procedure can be performed in the beach chair or lateral position, with the arm in traction. The beach chair position has the advantage of easy intraoperative adjustment of the arm for optimal exposure in the small joint space.

After assessment of range of motion under anesthesia, a standard posterior portal is established. It can be difficult to enter the joint due to contracture and capsular fibrosis, and cartilage lesions can occur. To facilitate introduction of the arthroscope and decrease the risk of iatrogenic injury, the surgeon can choose to inflate the joint space with saline using a spinal needle prior to introduction.

When visualization is achieved and anatomical landmarks are identified, an anterior portal is created in the rotator interval. A limited synovectomy is done using the shaver through a rigid cannula, taking care to avoid excess bleeding. Now the electrocautery device is introduced. It can be useful to remove the cannula because it restricts movement.

Capsular release is commenced in the rotator interval just anteroinferior to the biceps tendon. The capsule is released toward the edge of the subscapularis tendon. The release continues inferiorly down to the six o'clock position, staying anterior to the glenoid labrum. As release progresses, movement and visualization are gradually improved. When releasing the capsule in the axillary recess, it is important to be aware of the axillary nerve lying deep to these structures. At this point, the arthroscope is switched to the anterior portal, and the cautery device is placed in the posterior portal. The posterior capsule can now be addressed from posterosuperior to inferior and extralabral until a 360 degree release is completed. Additionally, the subacromial space is evaluated and any accompanying adhesions are released. Lastly, gentle manipulation is performed to evaluate the range of motion achieved. A few remaining contraction releases can be felt at this time.

Alternatively, selective release can be performed according to the portion of the contracted capsule specifically restricting range of motion. Circumferential release is usually performed. However, studies show no difference in long-term follow-up. ${ }^{53,54}$ Again, this intervention should be followed by physical exercises and management of pain to prevent stiffness.

Whether to combine the two interventions or perform capsular release only if MUA fails is not clear. ${ }^{55}$ Some surgeons prefer to do MUA prior to capsular release because it makes access to the shoulder with the arthroscope easier, preventing iatrogenic cartilage injury when creating the first portal. On the other hand, hemarthrosis can occur, resulting in obscured visualization and making capsular release more difficult.

De Carli et al compared arthroscopic arthrolysis in combination with MUA versus intra-articular steroid injections in 44 patients. ${ }^{56}$ The patients were diagnosed with frozen shoulder and had had symptoms for a minimum of 3 months. In the period before inclusion, the patients were treated with physical therapy and nonsteroidal anti-inflammatory agents. This treatment had not been satisfactory before entry into the study. They found better results within a period of 6 weeks in the MUA + arthroscopic release group, but no difference in long-term follow-up. However, it must be noted that, at the time of inclusion, patients had had symptoms for only 3 months and that the natural, self-limiting course could be the most important factor in terms of a good long-term outcome. On the other hand, this study showed good short-term results in the arthrolysis group, and supports this regimen for patients in whom more rapid rehabilitation is needed or concomitant procedures are required, eg, rotator cuff repair.

\section{Capsular distension}

Another treatment option is capsular distension, also known as hydrodilatation. The shoulder joint capsule can be distended by intra-articular infusion of fluid. Most commonly, injection of saline combined with an anesthetic or corticosteroid is performed. However, infusion with air and contrast fluid is also described. ${ }^{57-59}$ The theory behind this intervention is based on the pathophysiology of thickening and contraction of the joint capsule. This causes decreased intra-articular joint volume. The fluid is injected into the joint under pressure and will distend the joint volume by disrupting the adhesions and scar tissue. Some clinics use fluoroscopic guidance to ensure correct positioning of the needle. The 
expected effect of the procedure is increased intra-articular volume and thereby improvement of range of motion. The procedure can be performed under local anesthesia and takes about 15 minutes, after which the patient can leave the hospital immediately. However, some patients report that the procedure is very painful, and that the procedure is not suitable for the outpatient setting.

In a systematic review of five trials including 196 patients with frozen shoulder, capsular distension with saline and corticosteroids showed good short-term results with regard to pain reduction, with improved range of motion and function. Four of five of the studies reviewed had a high risk of bias, indicating that more research is necessary to determine whether capsular distension is better than alternative treatment strategies. Minor adverse effects included pain during and after the procedure. ${ }^{60}$ A prospective, randomized, double-blind, placebo-controlled trial in 48 patients compared intra-articular distension using a combination of saline and corticosteroids versus placebo. ${ }^{61}$ The short-term outcome was in favor of the capsular distension group. At 6-week and 12-week follow-up, the group treated by distension with saline and corticosteroids performed better than the placebo group. Gavant et al reported that 14 of 16 patients experienced immediate pain relief and increased range of motion after infiltration of $30 \mathrm{~mL}$ of a mixture of lidocaine, corticosteroids, and contrast media following arthrography. The good clinical results of this intervention were maintained for at least 6 months. ${ }^{59}$ In a prospective study, 22 patients underwent capsular distension followed by a physical therapy program. ${ }^{62}$ Prior to inclusion, all patients received 3 months of physical therapy. One year after intervention, 19 patients showed an improved range of motion. The three patients without significant improvement had more severe disease at the time of intervention. The major drawbacks of these studies are their small patient numbers and non-comparative nature.

In a 2008 trial, 76 patients were randomized to receive a corticosteroid injection and capsular distension or to corticosteroid injection alone. No difference in treatment effects was found between the groups at the 6-week follow-up. ${ }^{63}$ Capsular distension can be considered an option in patients with frozen shoulder refractory to physical therapy and corticosteroids. However, it should be individualized to meet specific patient needs.

\section{Postoperative rehabilitation}

A rigorous and early postoperative rehabilitation protocol is of the utmost importance to optimize the outcome of surgical management for frozen shoulder. This is done mainly in the outpatient setting since patients are usually discharged from the hospital on the first day after surgery.

Pain management is important in the early postoperative stage because disuse of the shoulder will bring stiffness. Physical therapy should be started directly after surgery to maintain the gained range of motion. It should be explained to the patient that compliance with the rehabilitation protocol will improve the clinical outcome. Frequent contact between the patient and physical therapist will encourage the patient to adhere to a home exercise protocol and ensure that the exercises are being performed correctly. Physical therapy should be continued for a prolonged period, not only until an acceptable range of motion is achieved. Maintaining the range of motion gained is also important. The exercises are basically the same as in the preoperative phase, as described above. Again, pain and vigorous exercises should be avoided, and stretching has to be done several times a day.

Some surgeons prefer inpatient postoperative care. Although the costs are higher and it is more convenient for the patient to be at home, maximal pain control is possible and supervised exercises can be done in the correct way. Further, patients can use shoulder immobilizers in order to maintain the glenohumeral joint in the desired position and prevent contractures, eg, an internal rotation-limiting sling or a humeral cuff during the night which forces the arm into abduction.

\section{Prevention}

Prevention of frozen shoulder would be preferable to treatment of the condition. However, there is no evidence supporting specific exercise programs to prevent frozen shoulder. Given the fact that frozen shoulder tends to occur in patients who have a shoulder that is immobilized for other reasons, like fracture or surgery, it seems reasonable that keeping the shoulder healthy and mobile can help in prevention of the condition. Early mobilization of the shoulder after injury or dislocation with adequate pain medication could therefore be a preventive measure. Despite the fact that the prevalence of frozen shoulder is higher in diabetic patients, a retrospective analysis of 201,513 diabetic patients showed no correlation between the prevalence of frozen shoulder and elevated glycated hemoglobin levels. ${ }^{64}$ Insulin-dependent patients are more likely to develop a frozen shoulder than non-insulin-dependent diabetic patients (odds ratio 1.93). ${ }^{64}$ Moreover, a normal glycated hemoglobin level will not prevent a frozen shoulder. Since there are no specific preventive measures, it is best to identify high-risk patients and minimize 
immobilization of the shoulder without compromising the outcome of other treatments for the shoulder.

\section{Conclusion}

In summary, frozen shoulder is a disabling disease and recovering is a long-term process. Many treatment options are available for the management of the frozen shoulder, but there is still no consensus in the literature regarding which therapeutic option is superior, mostly because of a lack of high-level evidence.

Given that a frozen shoulder is a benign, self-limiting condition, conservative treatment options are the first choice for management in the first stage of the disease. Supervised neglect can be combined with analgesia and stretching exercises as the pain subsides. ${ }^{7,65-67}$ Physical therapy and supervised home exercises, combined with pain management and intra-articular corticosteroid injections, are considered most important in the non-surgical treatment of frozen shoulder. Patient education and expectant management are very important features in managing patients with a frozen shoulder. Explanation of the pathophysiology and natural history to the patient will encourage acceptance of the condition and increase compliance with therapy. When this treatment is not satisfactory, and pain and loss of range of motion persists, invasive options can be considered, including arthroscopic capsular release combined with gentle MUA or capsular distension.

\section{Acknowledgment}

The authors would like to acknowledge Lotte Hulshof and Arianne van der Linden, from the Department of Physical Therapy, for providing clinical images of the stretching exercises.

\section{Disclosure}

The authors report no conflicts of interest in this work.

\section{References}

1. Reeves B. The natural history of the frozen shoulder syndrome. Scand J Rheumatol. 1975;4:193-196.

2. Binder AI, Bulgen DY, Hazleman BL, Roberts S. Frozen shoulder: a long-term prospective study. Ann Rheum Dis. 1984;43:361-364.

3. Hazleman BL. The painful stiff shoulder. Rheumatol Phys Med. 1972; 11:413-421.

4. Hakim AJ, Cherkas LF, Spector TD, MacGregor AJ. Genetic associations between frozen shoulder and tennis elbow: a female twin study. Rheumatology. 2003;42:739-742.

5. Hand C, Clipsham K, Rees JL, Carr AJ. Long-term outcome of frozen shoulder. J Shoulder Elbow Surg. 2008;17:231-236.

6. Levine WN, Kashyap CP, Bak SF, Ahmad CS, Blaine TA, Bigliani LU. Nonoperative management of idiopathic adhesive capsulitis. J Shoulder Elbow Surg. 2007;16:569-573.
7. Rizk TE, Pinals RS. Frozen shoulder. Semin Arthritis Rheum. 1982;11: 440-452.

8. Debeer P, Franssens F, Roosen I. Frozen shoulder and the Big Five personality traits. J Shoulder Elbow Surg. 2014;23:221-226.

9. Duplay S. De la periarthrite scapulo-humerale [On scapulo-humeral periarthritis]. Rev Pract D Trav De Med. 1896;53:226. French.

10. Codman EA. The Shoulder: Rupture of the Supraspinatus Tendon and Other Lesions in or About the Subacromial Bursa. Boston, MA, USA: Thomas Todd Company; 1934.

11. Neviaser JS. Adhesive capsulitis of the shoulder. J Bone Joint Surg Am. 1945;27:211-222.

12. Lundberg BJ. The frozen shoulder. Acta Orthop Scand. 1969; 119:1-59.

13. Bunker TD, Anthony PP. The pathology of frozen shoulder: a Dupuytren like disease. J Bone Joint Surg Br. 1995;77:677-683.

14. Uitvlugt G, Detrisac DA, Johnson LL, Austin MD, Johnson C. Arthroscopic observations before and after manipulation of frozen shoulder. Arthroscopy. 1993;9:181-185.

15. Wiley AM. Arthroscopic appearance of frozen shoulder. Arthroscopy. 1991;7:138-143.

16. Bunker TD, Lagas K, DeFerme A. Arthroscopy and manipulation in frozen shoulder. J Bone Joint Surg. 1994;76B Suppl 1:53.

17. Neer CS, Satterlee CC, Dalsey RM, Flatow EL. The anatomy and potential effects of contracture of the coracohumeral ligament. Clin Orthop. 1992;280:182-185.

18. Lequesne M, Dang N, Bensasson M, Mery C. Increased association of diabetes mellitus with capsulitis of the shoulder and shoulder-hand syndrome. Scand J Rheumatol. 1977;6:53-56.

19. Pal B, Anderson J, Dick WC, Griffiths ID. Limitation of joint mobility and shoulder capsulitis in insulin- and non-insulin-dependent diabetes mellitus. Br J Rheumatol. 1986;25:147-151.

20. Huang YP, Fann CY, Chiu YH, et al. Association of diabetes mellitus with the risk of developing adhesive capsulitis of the shoulder: a longitudinal population-based followup study. Arthritis Care Res (Hoboken). 2013;65:1197-1202.

21. Griggs SM, Ahn A, Green A. Idiopathic adhesive capsulitis. A prospective functional outcome study of nonoperative treatment. J Bone Joint Surg Am. 2000;82A:1398-1407.

22. Chambler AF, Carr AJ. The role of surgery in frozen shoulder. $J$ Bone Joint Surg. 2003;85:789-795.

23. Shaffer B, Tibone JE, Kerlan RK. Frozen shoulder. A long term follow up. J Bone Joint Surg Am. 1992;74:738-746.

24. Rundquist PJ, Anderson DD, Guanche CA. Shoulder kinematics in subjects with frozen shoulder. Arch Phys Med Rehabil. 2003;84:1473-1479.

25. Neviaser RJ, Neviaser TJ. The frozen shoulder: diagnosis and management. Clin Orthop Relat Res. 1987;223:59-64.

26. McLaughlin HL. On the frozen shoulder. Bull Hosp Joint Dis. 1951;12: 383-393.

27. Diercks RL, Stevens M. Gentle thawing of the frozen shoulder: a prospective study of supervised neglect versus intensive physical therapy in seventy-seven patients with frozen shoulder syndrome followed up for two years. Shoulder Elbow Surg. 2004;13:499-502.

28. Vermeulen HM, Stokdijk M, Eilers PH. Measurement of three dimensional shoulder movement patterns with an electromagnetic tracking device in patients with a frozen shoulder. Ann Rheum Dis. 2002;61:115-120.

29. Vermeulen HM, Obermann WR, Burger BJ. End-range mobilization techniques in adhesive capsulitis of the shoulder joint: a multiple-subject case report. Phys Ther. 2000;80:1204-1213.

30. Rookmoneea M, Dennis L, Brealey S. The effectiveness of interventions in the management of patients with primary frozen shoulder. $J$ Bone Joint Surg. 2010;92B:1267-1272.

31. Favejee MM, Huisstede BM, Koes BW. Frozen shoulder: the effectiveness of conservative and surgical interventions - systematic review. Br J Sports Med. 2011;45:49-56.

32. Buchbinder R, Green S, Youd JM, Johnston RV. Oral steroids for adhesive capsulitis. Cochrane Database Syst Rev. 2006;4:CD006189. 
33. Russell S, Jariwala A, Conlon R. A blinded, randomized, controlled trial assessing conservative management strategies for frozen shoulder. J Shoulder Elbow Surg. 2014;23:500-507.

34. Arroll B, Goodyear-Smith F. Corticosteroid injections for painful shoulder: a meta-analysis. Br J Gen Pract. 2005;55:224-228.

35. Lorbach O, Anagnostakos K, Scherf C, Seil R, Kohn D, Pape D. Nonoperative management of adhesive capsulitis of the shoulder: oral cortisone application versus intra-articular cortisone injections. J Shoulder Elbow Surg. 2010;19:172-179.

36. Shah N, Lewis M. Shoulder adhesive capsulitis: systematic review of randomised trials using multiple corticosteroid injections. $\mathrm{Br} \mathrm{J} \mathrm{Gen}$ Pract. 2007;57:662-667.

37. Bulgen DY, Binder Al, Hazleman BL, Dutton J, Roberts S. Frozen shoulder: prospective clinical study with an evaluation of three treatment regimens. Ann Rheum Dis. 1984;43:353-360.

38. Rizk TE, Pinals RS, Talaiver AS. Corticosteroid injections in adhesive capsulitis: investigation of their value and site. Arch Phys Med Rehabil. 1991;72:20-22.

39. Lee M, Haq AM, Wright V, Longton EB. Periarthritis of the shoulder: a controlled trial of physiotherapy. Physiotherapy. 1973;59:312-315.

40. Van derWindt DA, Koes BW, Deville W, BoekeAJ, de Jong BA, Bouter LM. Effectiveness of corticosteroid injections versus physiotherapy for treatment of painful stiff shoulder in primary care: randomised trial. BMJ. 1998;317:1292-1296.

41. Buchbinder R, Green S, Youd JM. Corticosteroid injections for shoulder pain. Cochrane Database Syst Rev. 2003;1:CD004016.

42. Neviaser AS, Hannafin JA. Adhesive capsulitis: a review of current treatment. Am J Sports Med. 2010;38:2346-2356.

43. Carette S, Moffet H, Tardif J, et al. Intra articular corticosteroids, supervised physiotherapy, or a combination of the two in the treatment of adhesive capsulitis of the shoulder: a placebo-controlled trial. Arthritis Rheum. 2003;48:829-838.

44. Jain TK, Sharma NK. The effectiveness of physiotherapeutic interventions in treatment of frozen shoulder/adhesive capsulitis: a systematic review. J Back Musculoskelet Rehabil. 2014;27:247-273.

45. Green S, Buchbinder R, Hetrick S. Acupuncture for shoulder pain. Cochrane Database Syst Rev. 2005;2:CD005319.

46. Dahan TH, Fortin L, Pelletier M, Petit M, Vadeboncoeur R, Suissa S. Double blind randomized clinical trial examining the efficacy of bupivacaine suprascapular nerve blocks in frozen shoulder. $J$ Rheumatol. 2000;27:1464-1469.

47. Neviaser RJ. Painful conditions affecting the shoulder. Clin Orthop. 1983;173:63-69.

48. Buchbinder R, Hoving JL, Green S, Hall S, Forbes A, Nash P. Short course prednisolone for adhesive capsulitis (frozen shoulder or stiff painful shoulder): a randomised, double blind, placebo controlled trial. Ann Rheum Dis. 2004;63:1460-1469.

49. Murnaghan JP. Frozen shoulder. In: Rockwood CA Jr, Matsen FA, editors. The Shoulder. Philadelphia, PA, USA: WB Saunders; 1990.

50. Iannotti JP. Manipulation under anesthesia did not enhance the benefit conferred by home exercises for frozen shoulder. J Bone Joint Surg. 2008;90A:1792.
51. Kivimäki J, Pohjolainen T, Malmivaara A, et al. Manipulation under anesthesia with home exercises versus home exercises alone in the treatment of frozen shoulder: a randomized, controlled trial with 125 patients. J Shoulder Elbow Surg. 2007;16:722-726.

52. Ogilvie-Harris DJ, Biggs DJ, Fitsialos DP, MacKay M. The resistant frozen shoulder: manipulation versus arthroscopic release. Clin Orthop. 1995;319:238-248.

53. Snow M, Boutros I, Funk L. Posterior arthroscopic capsular release in frozen shoulder. Arthroscopy. 2009;25:19-23.

54. Owens-Burkhart H. Management of frozen shoulder. In: Donatelli R, editor. Physical Therapy of the Shoulder. New York, NY, USA: Churchill Livingstone; 1991.

55. Chen J, Chen S, LiY, Hua Y, Li H. Is the extended release of the inferior glenohumeral ligament necessary for frozen shoulder? Arthroscopy. 2010;26:529-535.

56. De Carli A, Vadala A, Perugia D, et al. Shoulder adhesive capsulitis: manipulation and arthroscopic arthrolysus or intra-articular steroid injections? Int Orthop. 2012;36:101-106.

57. Ekelund A, Rydell N. Combination treatment for adhesive capsulitis of the shoulder. Clin Orthop Relat Res. 1992;282:105-109.

58. Fareed DO, Gallivan WR Jr. Office management of frozen shoulder syndrome: treatment with hydraulic distension under local anesthesia. Clin Orthop Relat Res. 1989;242:177-183.

59. Gavant ML, Rizk TE, Gold RE, Flick PA. Distention arthrography in the treatment of adhesive capsulitis of the shoulder. J Vasc Interv Radiol. 1994;5:305-308.

60. Buchbinder R, Green S, Youd JM. Arthrographic distension for adhesive capsulitis (frozen shoulder). Cochrane Database Syst Rev. 2008;1:CD007005.

61. Buchbinder R, Green S, Forbes A. Arthrographic joint distension with saline and steroid improves function and reduces pain in patients with painful stiff shoulder: results of a randomized, double blind, placebo controlled trial. Ann Rheum Dis. 2004;63:302-309.

62. Vad VB, Sakalkale D, Warren RF. The role of capsular distension in adhesive capsulitis. Arch Phys Med Rehabil. 2003;84:1290-1292.

63. Tveitå EK, Tariq R, Sesseng S. Hydrodilatation, corticosteroids and adhesive capsulitis: a randomized controlled trial. BMC Musculoskelet Disord. 2008;9:53.

64. Yian EH, Contreras R, Sodl JF. Effects of glycemic control on prevalence of diabetic frozen shoulder. J Bone Joint Surg Am. 2012;94: 919-923.

65. Placzek JD, Roubal PJ, Freeman DC, Kulig K, Nasser S, Pagett BT. Long-term effectiveness of translational manipulation for adhesive capsulitis. Clin Orthop Relat Res. 1998;356:181-191.

66. Green S, Buchbinder R, Hetrick S. Physiotherapy interventions for shoulder pain. Cochrane Database Syst Rev. 2003;2:CD004258.

67. Yoon SH, Lee HY, Lee HJ, Kwack KS. Optimal dose of intraarticular corticosteroids for adhesive capsulitis: a randomized, triple-blind, placebo-controlled trial. Am J Sports Med. 2013;41: 1133-1139.
Orthopedic Research and Reviews

\section{Publish your work in this journal}

Orthopedic Research and Reviews is an international, peer-reviewed, open access journal focusing on the patho-physiology of the musculoskeletal system, trauma, surgery and other corrective interventions to restore mobility and function. Advances in new technologies, materials, techniques and pharmacological agents are particularly welcome. The journal welcomes Submit your manuscript here: http://www.dovepress.com/orthopedic-research-and-reviews-journa

\section{Dovepress}

original research, clinical studies, reviews \& evaluations, expert opinion and commentary, case reports and extended reports. The manuscript management system is completely online and includes a very quick and fair peer-review system, which is all easy to use. Visit http://www.dovepress. com/testimonials.php to read real quotes from published authors. 